\title{
Performance test in semispherical solar collectors with discontinuous absorber
}

\author{
Carlos Armenta-Deu* \\ Facultad de Ciencias Físicas. Universidad Complutense de Madrid, 28040 Madrid, Spain
}

\section{A R T I C L E I N F O}

\section{Article history:}

Received 14 November 2018

Received in revised form

25 March 2019

Accepted 25 April 2019

Available online 3 May 2019

Keywords:

Semispherical collector

Thermal performance

\begin{abstract}
A B S T R A C T
This work aims at characterizing the performance of a solar thermal collector with non-continuous absorber area. The study bases its development on the classical performance method for flat-plate collectors, but using the effective absorber area that intercepts solar radiation. The work determines not only thermal efficiency of the solar collector but corrected overall thermal losses coefficient for the specific geometric design.
\end{abstract}

() 2019 Elsevier Ltd. All rights reserved.

\section{Introduction}

Solar thermal collectors receive solar radiation from any direction, but the contribution to the absorbed energy depends on the type of radiation, direct, diffuse or reflected, and on the effective area covered by each type onto the absorber surface. Therefore, solar radiation does not strike the absorber at normal incidence but for the noontime. This situation provokes energy losses that increase with the incidence angle. To avoid this inconvenience, the solar collector must track the Sun, mounting a tracking system what complicates the design and increases the cost. A practical solution is redesigning the absorber geometry so it can receive sunrays at normal incidence at any time; the resulting geometry is a sphere. Because lower half-section of a sphere receives low solar radiation compared to the upper one, designers have evolved the absorber geometry to a semispherical dome. The new geometry reduces size and cost.

In present days flat-plate solar collectors share low thermal applications such as domestic water with new designs like spherical or semispherical collectors. These new devices look for increasing solar energy collection without the need of a tracking system, a typical characteristic of higher temperature applications, but not applicable to low temperature systems because of the high price and low cost effectiveness.

This new geometry is also very convenient for any type of

\footnotetext{
* Corresponding author.

E-mail address: cardeu@fis.ucm.es.
}

location, low or high latitude, but it is especially interesting in countries of high latitude where sun altitude is very low in most of the year making the use of flat-plate collectors insufficient to receive the required amount of solar radiation. Some studies [1] demonstrates that the energy collected in semispherical solar devices is higher than in flat-plate collectors, especially in the aforementioned areas. Besides, the yearly energy productivity for these new collectors result in higher values than in the case of flat-plate collectors.

Previous studies on effectiveness in semispherical solar collectors [2] have proved these systems produce a $37 \%$ more energy than equivalent surface flat-plate collectors; even more, the results show that energy gain in this type of collectors are comparable with the obtained in vacuum tube ones [3]. These studies, however, are made on continuous absorber, using the front semispherical geometry surface for solar radiation interception and avoiding the effect of background reflection on soil. Therefore, the effective area that intercepts solar radiation is not the same as in discontinuous absorber, the focus of our study, and the performance curve results in different values, especially what concerns the slope of the curve, thus, the global thermal losses coefficient. Consequently, the existing performance curve for continuous absorber in semispherical solar collectors is not applicable to semispherical collectors with discontinuous absorber.

Solar thermal collectors performance has been currently determined using the classical expression developed for flat-plate collectors [4], now using the recently approved methodology [5]. This new Standard unifies all the former American, European and 


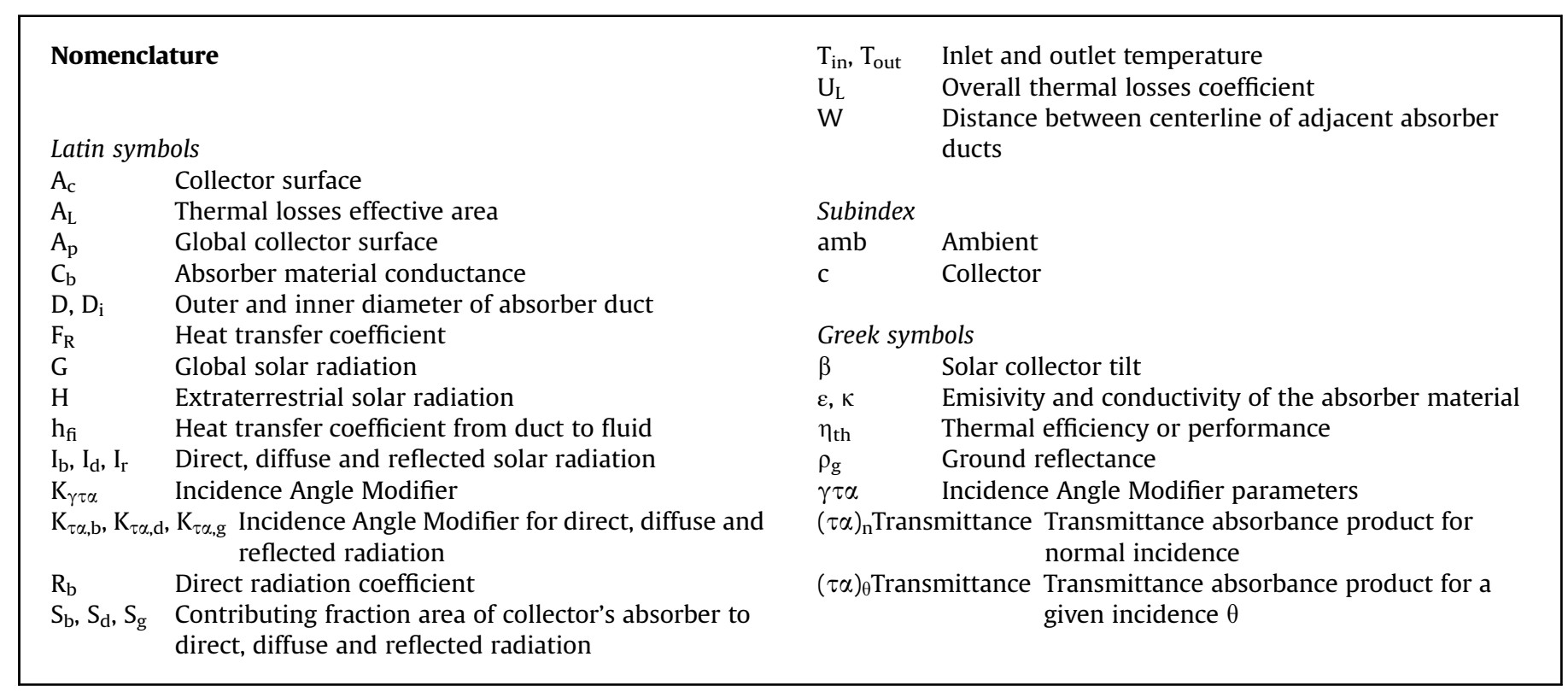

International Standards. However, some solar collector types still do not fit to the Standard testing methodology, in particular solar collectors with variable-geometry [6]. Besides, in non-flat-plate collectors like spherical or semi-spherical ones, the solar radiation intercepted area by the absorber does not match with geometrical surface [7], resulting in a deviation that produces errors in the performance determination [8] as well as in overall thermal losses calculation [9].

Performance of flat plate collectors relates useful energy to the incoming solar radiation. Because solar radiation accounts for direct and diffuse components, the effects of the angle of incidence should be taken into account, what is included in the mathematical expression for the received radiation through the Incidence Angle Modifier (IAM) [10,11]. In spherical or semispherical solar collectors, the influence of the angle of incidence of the solar radiation is different because a fraction of the collector's absorber receives solar radiation at normal incidence during all solar daytime. Therefore, the procedure used for flat plate collectors may introduce errors in the determination of the collector's performance. Moreover, useful energy is computed as the difference between absorbed solar energy and thermal losses. Due to the difference between geometrical and absorbing area in spherical or semispherical solar collectors, thermal losses cannot be computed as in flat plate collectors, thus affecting the classical methodology or the new approved one [4,5]. Nevertheless, thermal performance in spherical or semi-spherical collectors can be done using the ISO norm [5], but adjusting the expression to the specific collector's geometry. Because of the different area covered by direct and diffuse radiation in flat plate and spherical or semispherical collectors, the parameter that must be adjusted in this latter case is the effective area for both, direct and diffuse component, considering the effective area is the fraction for which the collector intercepts a solar radiation component.

\section{Theoretical basis}

The best way to determine thermal performance of any device, such as solar collectors, is through the classical thermodynamic efficiency. Mathematically: $\eta_{t h}=\dot{Q}_{u} / P_{i n}$

where $\dot{Q}_{u}$ accounts for useful energy rate and $P_{i n}$ for incoming power. Applying this expression to a solar collector, we have:

$\eta_{\text {th }}=\frac{\dot{Q}_{u}}{G S}=\frac{\dot{m} c\left(T_{\text {out }}-T_{\text {in }}\right)}{G S}=1-\frac{U_{L}\left(T_{c}-T_{\text {amb }}\right)}{G}$

Which is the theoretical expression for thermal performance determination.

This expression however, is modified because solar collector performance does not reach $100 \%$ in real conditions, as the trasmitance-absorbance product is lower than 1 ; therefore, eq. (2a) should be transformed into the next one:

$\eta_{t h}=(\tau \alpha)-\frac{U_{L}\left(T_{c}-T_{a m b}\right)}{G}$

This expression, however, cannot be applied in its present form to spherical or semispherical collectors, as the receiving surface is not the same as the emitting one. Therefore, equation (2) should be transformed into the following:

$\eta_{t h}=\frac{\int \dot{Q}_{u} d t}{\int\left(S_{b} I_{b}+S_{d} I_{d}+S_{r} I_{r}\right) d t}$

where $I_{b}, I_{d}$ and $I_{r}$ account for direct, diffuse and reflected radiation, and $S_{b}, S_{d}$ and $S_{r}$ are the corresponding fraction of the absorber area that intercepts direct, diffuse or reflected radiation, respectively.

As thermal performance is currently given as an instantaneous value, we can express equation (3a) under the form:

$\eta_{t h}=\frac{\dot{Q}_{u}}{S_{b} I_{b}+S_{d} I_{d}+S_{r} I_{r}}$

Now considering direct, diffuse and reflected component do not strike the absorber with the same angle of incidence, we must 
include the effect of this factor into the efficiency expression; therefore:

$\eta_{t h}=\frac{\dot{Q}_{u}}{G S_{e f}}$

where $\mathrm{GS}_{\mathrm{ef}}$ is the effective solar radiation power taking into account the specific contribution of each component. The term $\mathrm{GS}_{\mathrm{ef}}$ is expressed as:

$$
\begin{aligned}
& G S_{e f}=\left[I_{b} R_{b} K_{\tau \alpha, b}(\tau \alpha)_{n}\right] S_{b}+\left[I_{d} K_{\tau \alpha, d}(\tau \alpha)_{n}\left(\frac{1+\cos \beta}{2}\right)\right] S_{d} \\
& +\left[\rho_{g}\left(I_{b}+I_{d}\right) K_{\tau \alpha, g}(\tau \alpha)_{n}\left(\frac{1-\cos \beta}{2}\right)\right] S_{g}
\end{aligned}
$$

where $K_{j}$ is the Incidence Angle Modifier for each component. The $(\tau \alpha)_{\mathrm{n}}$ coefficient accounts for the effects of glass cover transmittance and receiver absorbance on the solar radiation at normal incidence, and is currently given by the manufacturer. The radiation coefficient, $R_{b}$, is usually obtained from the expression

$R_{b}=\left(H_{o \beta} / H_{o}\right)$

where $\mathrm{H}_{\mathrm{o} \beta}$ accounts for extraterrestrial solar radiation onto tilted plane and $\mathrm{H}_{\mathrm{o}}$ refers to the horizontal [12]. Specific area contributions for direct, diffuse and reflected radiation can be obtained from the proposed mathematical model [13].

Useful energy can be obtained subtracting thermal losses from energy gains, considering the energy received and thermal losses correspond to a given area, $\mathrm{A}_{c}$. In such conditions, thermal performance is expressed as:

$\eta_{t h}=\frac{F_{R}(\tau \alpha)_{\theta} G S_{e f}-U_{L} A_{c}\left(T_{c}-T_{a m b}\right)}{G S_{e f}}=F_{R}(\tau \alpha)_{\theta}-\frac{U_{L} A_{c}\left(T_{c}-T_{a m b}\right)}{G S_{e f}}$

where the first term is the effective energy transfer from solar radiation to the absorber while second one refers to thermal losses. The $F_{R}$ factor is the heat transfer coefficient and $(\tau \alpha)_{\theta}$ accounts is the transmittance-absorbance product for a given angle of incidence, $\theta$. Equation (7) takes into account the effective trasmitanceabsorbance product, $\tau \alpha$, from equation (2b) is affected by the thermal transfer efficiency through the $F_{R}$ coefficient, so $(\tau \alpha)=F_{R}$ $(\tau \alpha)_{\theta}$. On the other hand, the $(\tau \alpha)_{\theta}$ coefficient is difficult to get in direct way, so we use the alternative expression:

$(\tau \alpha)_{\theta}=(\tau \alpha)_{n} K(\theta)=(\tau \alpha)_{n}\left[1-b_{o}\left(\frac{1}{\cos \theta_{i}}-1\right)\right]$

Being $\theta_{\mathrm{i}}$ the angle of incidence of the solar radiation and $b_{0} a$ parameter depending on the number of glass covers.

Heat transfer coefficient is also difficult to determine as it depends on collector's geometry and type of materials. Some authors have proposed the following equation for the $F_{R}$ factor [14]:

$F_{R}=\frac{\dot{m} c}{A_{C} U_{L}}\left[1-\exp \left(-\frac{A_{C} U_{L} F^{\prime}}{\dot{m} c}\right)\right]$

where the coefficient $\mathrm{F}^{\prime}$ is obtained from the expression:

$F^{\prime}=\frac{1 / U_{L}}{W\left[\frac{1}{U_{L}[D+(W-D) F]}+\frac{1}{C_{b}}+\frac{1}{\pi D_{i} h_{f_{i}}}\right]}$
The $\mathrm{F}$ coefficient is the efficiency factor of heat transfer from plate to duct, and given by:

$F=\tanh \left[\frac{f(W-D)}{2}\right] / \frac{f(W-D)}{2}$

Being $\mathrm{f}$ an empirical parameter factor depending on absorber material conductivity. In semispherical collectors, because the duct plays the role of a plate, $\mathrm{F}=1$.

The $A_{c}$ parameter in eq. (9) is called "collector's area" although it does not correspond to global area because it represents the absorber fraction that intercepts solar radiation.

On the other hand, the $U_{L}$ coefficient computes conduction, convection and radiation thermal transfer from collector to surroundings. Considering constant temperature in the absorber overall thermal losses can be calculated from the general expression:

$\dot{Q}_{L}=A_{c}\left[\frac{\kappa}{e}\left(T_{c}-T_{a m b}\right)+h\left(T_{c}-T_{a m b}\right)+\sigma \varepsilon\left(T_{c}^{4}-T_{s k y}^{4}\right)\right]$

where $\kappa$ is the thermal conductivity of the absorber material, $e$ the thickness of the absorber wall, $h$ the convection coefficient, and $\varepsilon$ the absorber emissivity. Because $\mathrm{T}_{\text {sky }}$ is difficult to establish, we consider the absorber is emitting to the environment, therefore, $\mathrm{T}_{\text {sky }}=\mathrm{T}_{\text {env. }}$ Comparing the second term in eq. (7) and eq. (12), and developing the radiative emission term in eq. (12), it results:

$U_{L}=\frac{\kappa}{e}+h+4 \sigma \varepsilon T_{c}^{3}$

An expression that allows to determine the overall thermal losses coefficient as a function of the collector's temperature (see Fig. 1).

\section{Solar radiation onto COLLECTOR'S absorber}

A previous work [15] has developed a function to determine the effective area of a semi-spherical solar collector that intercepts direct radiation. This work considers the absorber as a group of semicircular stripes of reducing diameter from bottom to top, representing the spiral rolled up cylindrical duct (see Fig. 3), in

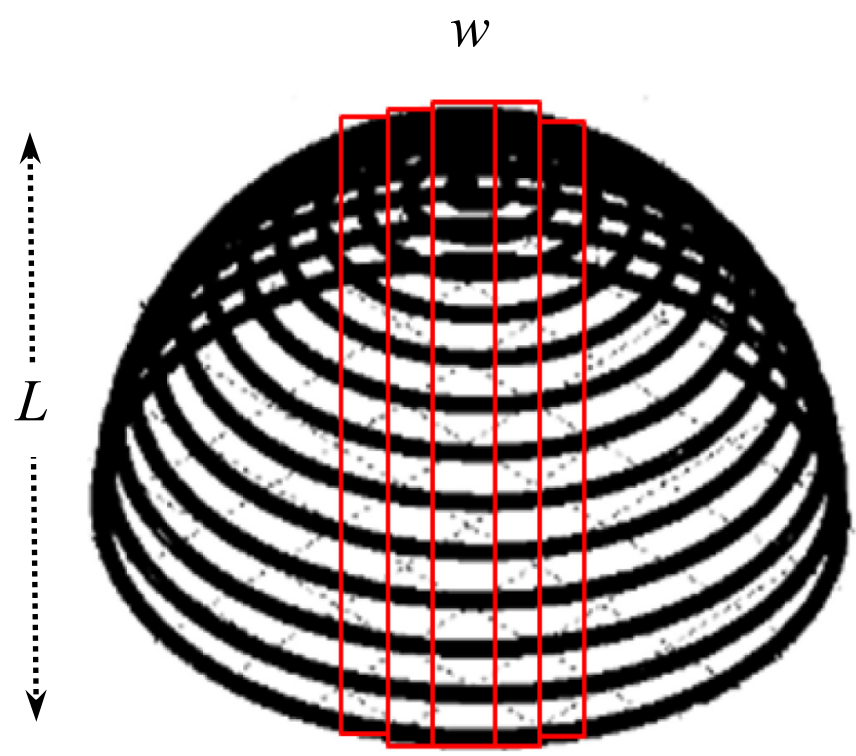

Fig. 1. Schematic representation of the absorber. 


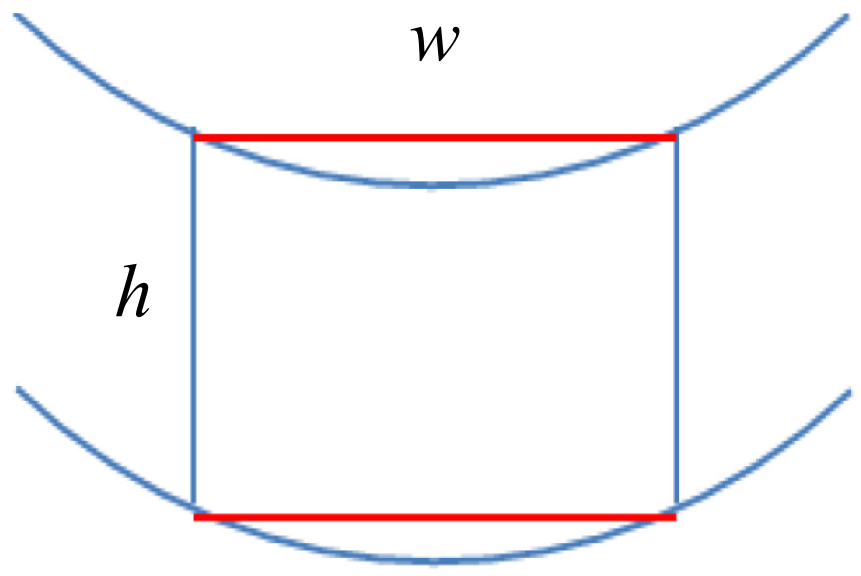

Fig. 2. Geometrical approximation of the absorber section.

which the mathematical model determines the solar radiation striking onto a section of every stripe of width, $w$, and height, $h$. Because $\mathrm{w}$ is very small, a geometrical approximation to a rectangle is taken into account (see Fig. 4). In this particular representation, $w$ is the circular segment fraction subtended for a $\lambda$-angle, being

$w=2\left[R_{n} / \cos (n \lambda)\right] \sin (\lambda / 2)$

where $R_{n}$ is the radius of the considered circular stripe. Therefore, the intercepted solar radiation for any stripe is:

$I_{n}=I_{C} \cos n \lambda$

being $n$ the number of section in every stripe and $I_{c}$ the solar radiation at normal incidence.

Now considering the stripes are regularly distributed from bottom to top, we can define the gap between two consecutive stripes as $\alpha$ (see Fig. 5), where the $\alpha$-value for any stripe is given by the expression [4]:

$\alpha_{n}=\frac{180 \alpha}{180-2 n \lambda}$

Adding all contributions we obtain the global intercepted area

$A=\sum_{n} \frac{\pi}{2} w_{n} F_{n}$

where $F_{n}$ is the interception factor defined as:

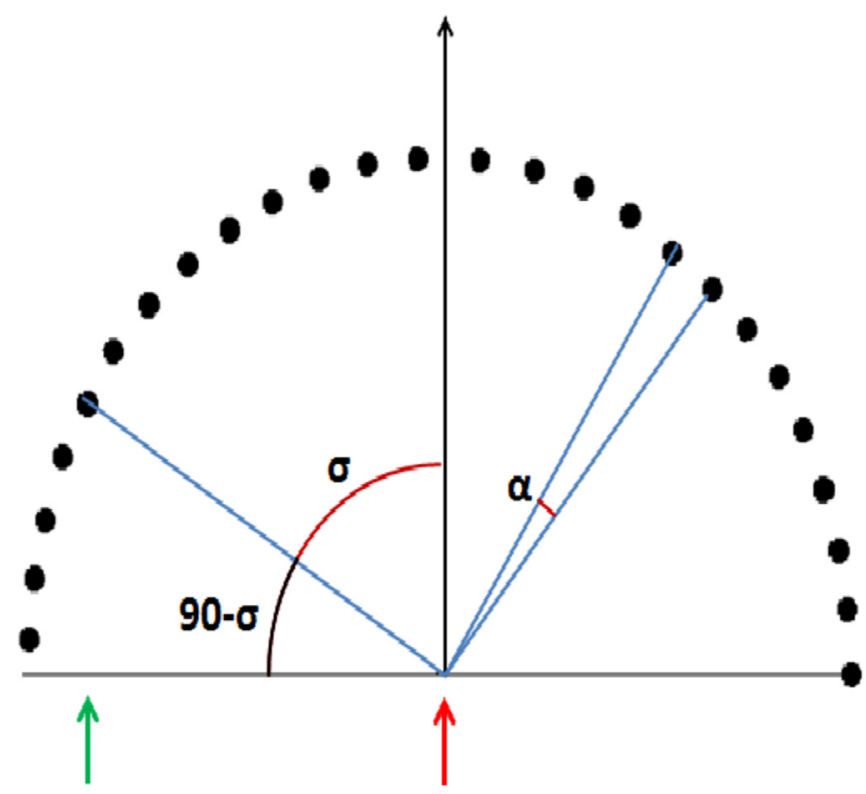

Fig. 3. Absorber frontal cross-section distribution.

$H(x)=\mid \begin{aligned} & 0 \rightarrow x<0 \\ & 1 \rightarrow x>0\end{aligned}$

We tested a commercial semispherical solar collector from the Bubblesun Company [16], made up of a rugged polyethylene cylindrical duct of $8 \mathrm{~mm}$ diameter, spirally rolled, covered by a methacrylate transparent dome (see Fig. 4). The structure stays on a polyurethane squared base covered by a high reflective aluminum foil. Heat transfer fluid (water) enters the absorber through the bottom side and exits at the opposite side. To assure the validity of the experimental results, and to validate the proposed methodology for thermal performance test in semispherical solar collectors, we used twin units. The units were placed on a horizontal stand, next to each other, exposed to solar $\{\{\}\}$ radiation, and connected to a data acquisition system to register flow rate, temperatures and solar radiation. Temperature sensors of K-type thermocouple were inserted at the inlet and outlet connections, as well as in a water tank placed behind the collectors and connected to them through a pump. The ambient temperature sensor is placed in a meteorological cabin. An optical device connected to the data acquisition system measures water flow. To determine solar radiation striking

$F_{n}=\left[R_{n} / \cos (n \lambda)\right] \cos (n \lambda)\left(1-\cos \theta_{i}\right)+D \frac{90-\theta_{n}}{n \lambda}+\left[H\left(\theta_{n}-\theta_{i}\right) D \frac{90-\theta_{n}}{n \lambda}\right]++\left[H\left(\theta_{n}-\theta_{i}\right) D \frac{90-\theta_{i}}{n \lambda}\right]+\left[H\left(\theta_{n}-\theta_{i}\right) R \cos (n \lambda)\left(\cos \theta_{i}-\cos \theta_{n}\right)\right]$

Where $\theta_{\mathrm{i}}$ is the solar radiation incidence angle, $\theta_{\mathrm{n}}$ the solar radiation angle of incidence for any particular stripe, and $\mathrm{D}$ is the diameter of the duct, which can be expressed in terms of geometrical parameters as

$D=R_{n}\left[\cos \theta_{n}-\cos \left(\theta_{n}+\alpha_{n}\right)\right]$

$\mathrm{H}$ is the well-known Heaviside function onto the collectors, two identical solar meters were placed on the stand platform of each collector, measuring global horizontal radiation. We checked the solar meters were not shadowed by the collector's structure at any time.

To measure diffuse radiation, a third solar meter was placed on the side of the collectors, onto a horizontal stand, with a shadowing ring. A view of the experimental device is shown in Fig. 5, with the corresponding layout in Fig. 6. 


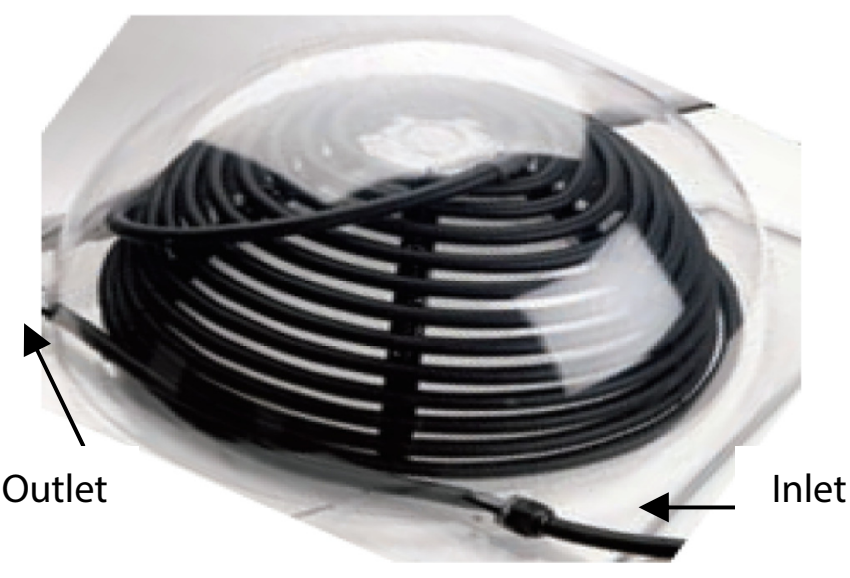

Fig. 4. Semispherical solar collector.

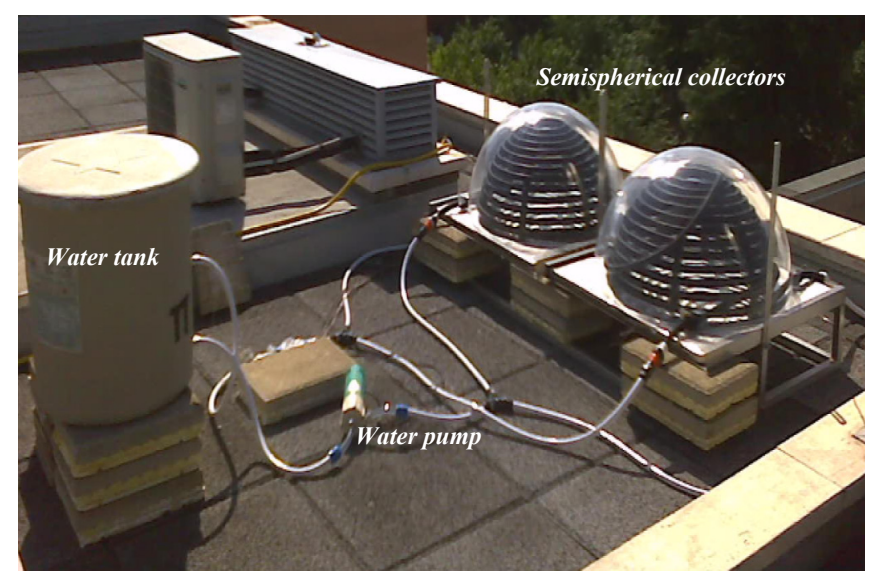

Fig. 5. View of experimental device.

Voltage regulator is connected to the system to modify the supplied power to the water pump, thus varying the flow rate; this allows testing the system under variable flow rates. Flow meter operates under CD current, what requires a battery to supply continuous energy; battery is recharged from network trough an $\mathrm{AC} / \mathrm{DC}$ converter. The optical system to measure flow rate consists of a frequency meter that detects the frequency of the optical signal and sends it to a measuring device connected to the PC and data logger. Water tank is used only in open loop tests while in closed loops it is by-passed.

\section{Experimental tests}

We run tests on clear sky day from early morning to late afternoon. Global and diffuse solar radiation onto horizontal plane was measured using two solar meters SKYE SK1110 within accuracy of $1 \%$, recording data every $5 \mathrm{~min}$. Solar radiation data are shown in Fig. 7, where continuous and dashed lines account for global and diffuse radiation, respectively. Flow rate was set up at the maximum value of $25 \mathrm{l} / \mathrm{min}$., using a centrifugal pump, HAMAMATSU PR210, that allows an accuracy of $0.1 \mathrm{l} / \mathrm{min}$. Inlet and outlet collector temperatures were measured at regular intervals, using Ttype thermocouple within accuracy of $0.1^{\circ} \mathrm{C}$, and recorded at the same time interval as solar radiation. Fig. 8 shows the evolution of collector's temperatures during test.

Applying conventional methodology for solar collector's thermal performance, and considering effective and geometrical

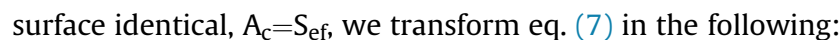

$\eta_{t h}=F_{R}(\tau \alpha)_{\theta}-U_{L} \frac{\left(T_{c}-T_{a m b}\right)}{G}=F_{R}(\tau \alpha)_{\theta}-U_{L} T_{e q}$

To determine thermal performance instantaneous measurements are used for ambient, inlet and outlet temperature as well as for solar radiation. Performance is obtained as an energy balance between incoming solar energy and outlet energy from the collector, according to the relation:

$\eta=\frac{\dot{m} c\left(T_{o}-T_{i}\right)}{G S_{e f f}}$

Being $\dot{m}$ the flow rate, $\mathrm{c}$ the specific heat of the heat carrier, water in our case, $T_{0}$ and $T_{i}$ collector's outlet and inlet temperature, $G$ the solar radiation onto tilted plane, and $\mathrm{S}_{\text {eff }}$ the effective absorber area of the solar collector.

The performance was calculated using the projection of the frontal cross section exposed to solar radiation as the absorbent surface. In such conditions, we obtained the following results (see Fig. 9).

We notice thermal performance matches the expected behavior, following a linear dependence on equivalent temperature, with optical efficiency of $82 \%$ for both collectors. Applying eq. (7), we have $\mathrm{F}_{\mathrm{R}}(\tau \alpha)_{\theta}=0.82$, and using eq. (8), and determining $K(\theta)$ (see Fig. 10), we have:

$(\tau \alpha)_{\theta}=0.86 \rightarrow F_{R}=0.96$

This is coherent with current values for solar collectors.

Using error analysis [17] and based on data uncertainty, we can establish the uncertainty in determining the equivalent temperature, $T_{\text {eq }}$, resulting in a value between $\pm 0.03( \pm 3) \%$ and $\pm 0.05( \pm 5 \%)$, depending on the operating zone. The uncertainty associated to the $(\tau \alpha)$ value is of \pm 0.05 ( $\pm 5 \%$ ) according to data provided by the collector's manufacturer.

Now taking into account eq. (22), an applying error analysis to this expression, and considering the uncertainty of the parameters involved in the equation, we can obtain global propagation uncertainty according to the expression:

$\frac{\delta \eta}{|\eta|}=\sqrt{\left(\frac{\delta \dot{m}}{\dot{m}}\right)^{2}+\left(\frac{\delta c}{c}\right)^{2}+\left(\frac{\delta T_{o}}{T_{o}}\right)^{2}+\left(\frac{\delta T_{i}}{T_{i}}\right)^{2}+\left(\frac{\delta G}{G}\right)^{2}+\left(\frac{\delta S_{\text {eff }}}{S_{\text {eff }}}\right)^{2}}$

Which is always lower to the value given by the expression:

$\frac{\delta \eta}{|\eta|}=\frac{\delta \dot{m}}{|\dot{m}|}+\frac{\delta c}{|c|}+\frac{\delta T_{o}}{\left|T_{o}\right|}+\frac{\delta T_{i}}{\left|T_{i}\right|}+\frac{\delta G}{|G|}+\frac{\delta S_{\text {eff }}}{\left|S_{\text {eff }}\right|}$

If we assume the maximum uncertainty, according to expression (23b), the uncertainty calculation produces the following result:

$$
\begin{aligned}
\frac{\delta \eta}{|\eta|} & =\frac{\delta \dot{m}}{|\dot{m}|}+\frac{\delta c}{|c|}+\frac{\delta T_{o}}{\left|T_{o}\right|}+\frac{\delta T_{i}}{\left|T_{i}\right|}+\frac{\delta G}{|G|}+\frac{\delta S_{\text {eff }}}{\left|S_{\text {eff }}\right|} \\
& = \pm(0.004+0.001+0.02+0.02+0.001+0.025)= \pm 0.071
\end{aligned}
$$

These results indicate the classical methodology can be initially applied to spherical or semispherical collectors using the frontal cross section exposed to solar radiation as the absorbent surface, as in the case of flat plate ones. However, comparing results for both collectors, we appreciate the slope of the straight line differs from 


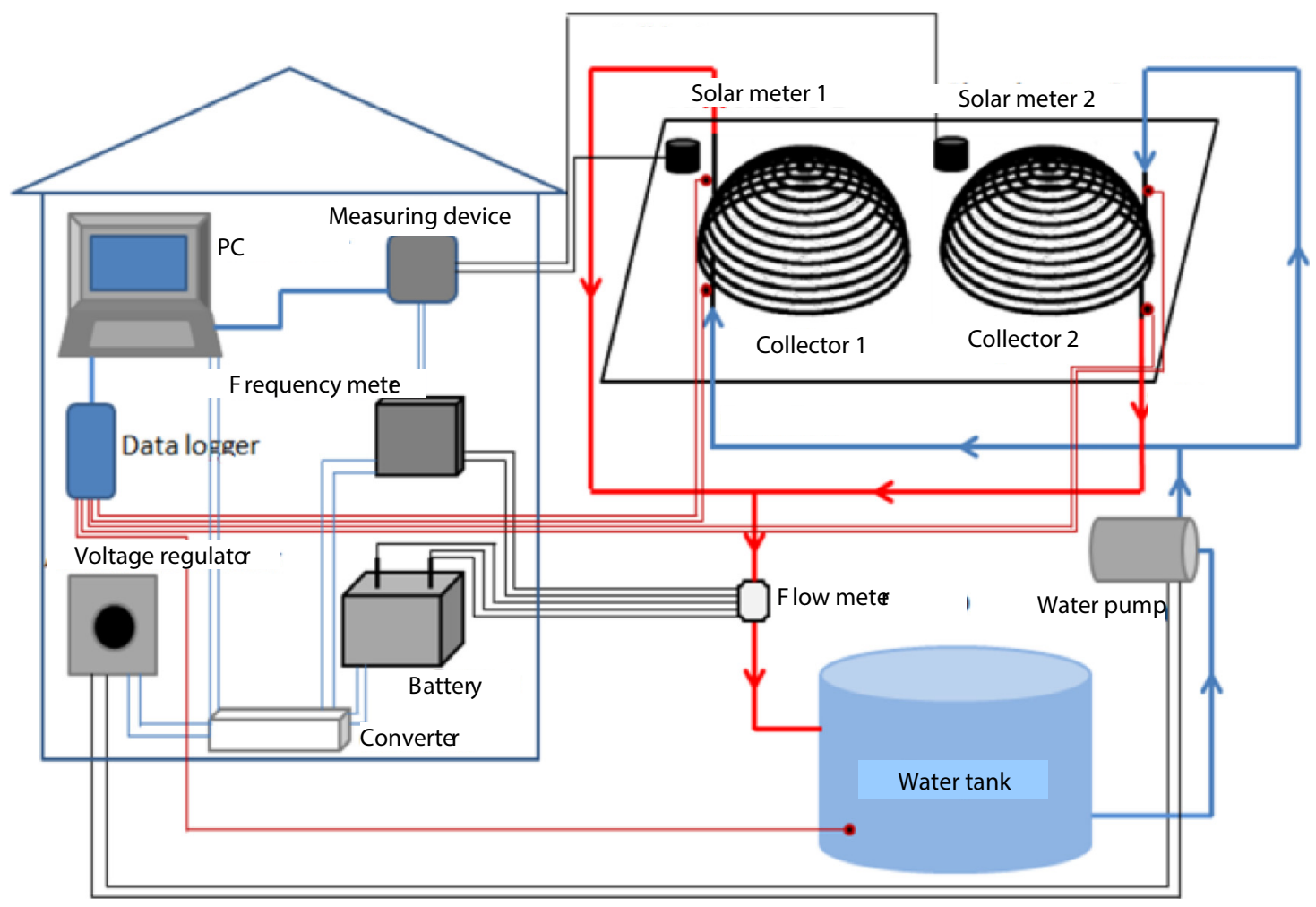

Fig. 6. Layout of experimental device.

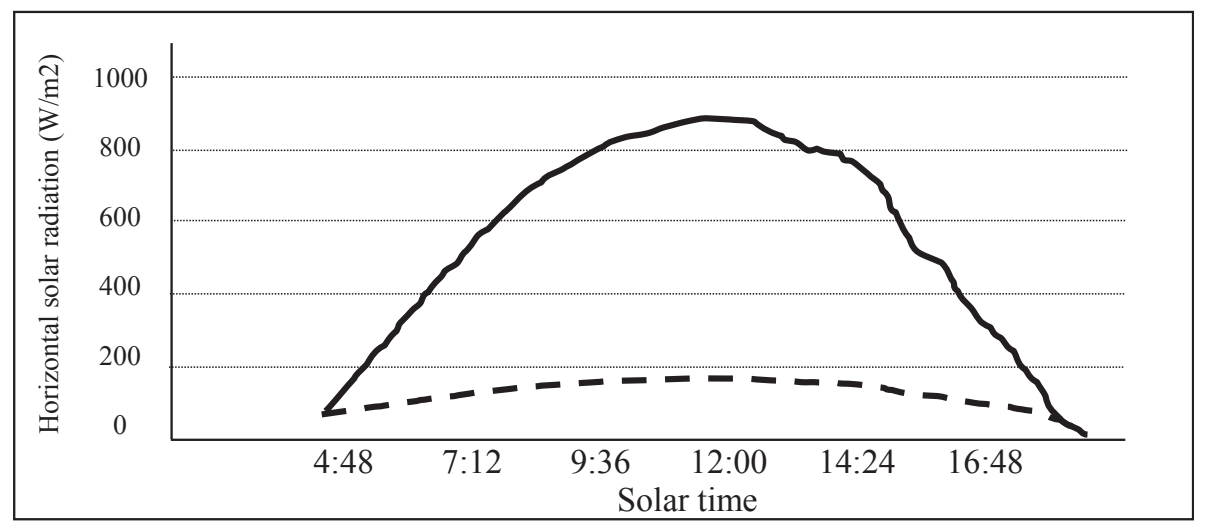

Fig. 7. Solar radiation evolution on test day.

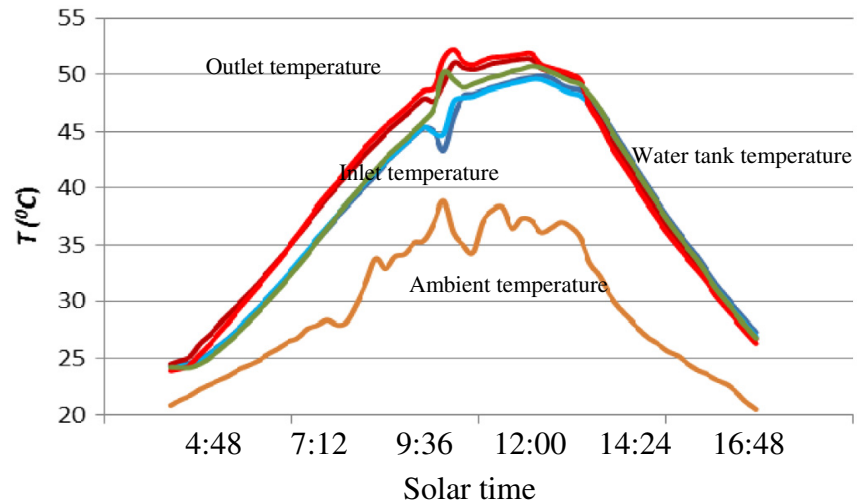

Fig. 8. Collector temperature evolution on test day. one collector to the other, what indicates overall thermal losses is different for both collectors. To solve this apparent anomaly, performance test was repeated under different operational conditions, obtaining the following results:

The second group of tests shows a closer agreement in the slope between both collectors, although optical performance for the second one slightly differs. Because there is not a unique value for the key parameters, $\mathrm{U}_{\mathrm{L}}$ and $(\tau \alpha)_{\mathrm{o}}$, we averaged the values for the two collectors and tests, excluding the optical efficiency for the collector $\mathrm{n}^{\circ} 2$ at the second test, due to its inconsistency to the other three values, which are almost identical. The result of this averaging process is $\mathrm{UL}=4.87 \mathrm{~W} / \mathrm{m}^{2} \cdot \mathrm{K}$ and $(\tau \alpha)_{0}=0.8248$. From these data, we can establish the performance equation for the semispherical collector as:

$\eta_{t h}=-4.87 T_{e q}+0.8248$ 

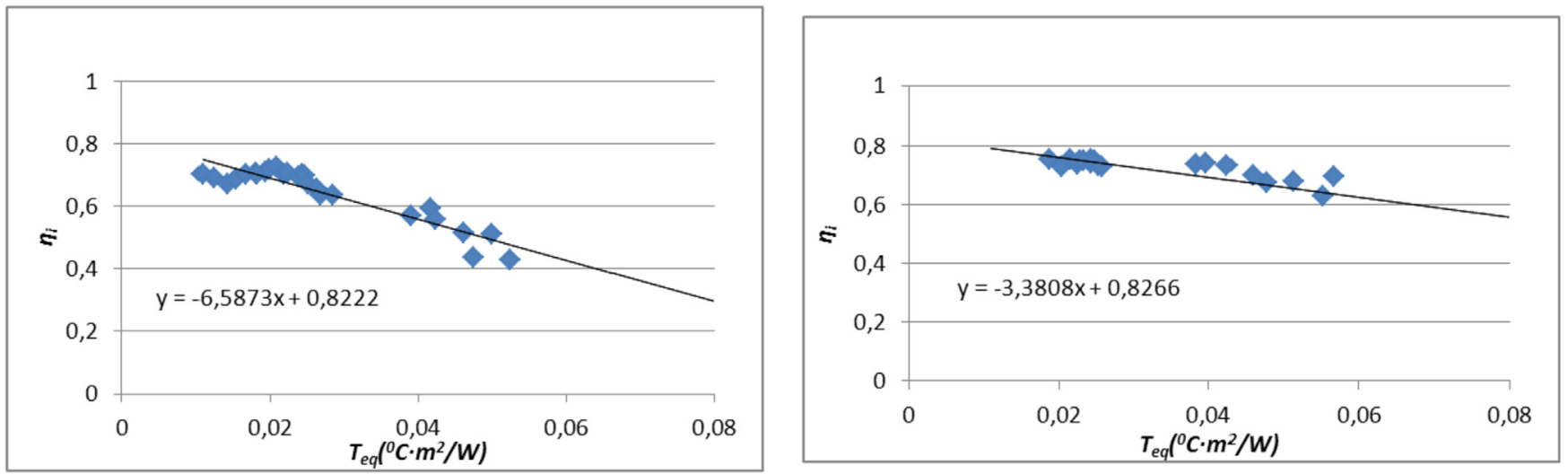

Fig. 9. a. Thermal performance of semispherical solar collector (1st test): collector 1, (b) Thermal performance of semispherical solar collector (1st test): collector 2 .

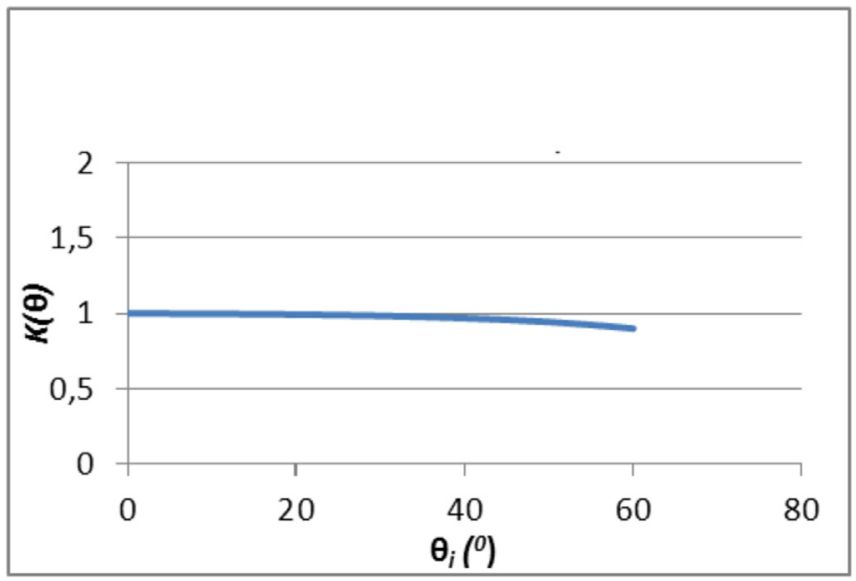

Fig. 10. Incidence angle modifier.

The so obtained values are very coherent with a conventional solar collector, indicating the measuring values are correct (see Fig. 11). Nevertheless, if we look at technical data provided by the manufacturer (Fig. 12), we observe that performance curve does not match with experimental results. In fact, optical efficiency is about $100 \%$, which is not very consistent with real conditions; besides, if we calculate the slope of the curve, through the straight trending line, we obtain a $U_{L}$ value of $16.75 \mathrm{~W} / \mathrm{m}^{2} \cdot \mathrm{K}$, much higher than the one obtained in our tests (see Fig. 2).

To solve the discrepancy we propose the use of a new definition

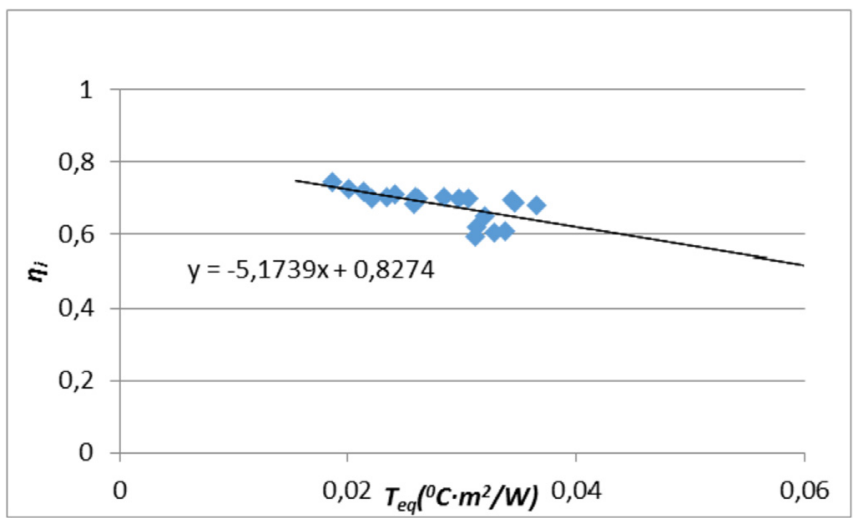

for the "equivalent temperature", $T_{e q}$, considering the thermal losses effective area and the absorbing area are different. Mathematically:

$T_{e q}^{o}=\frac{T_{i n}-T_{a m b}}{G} \frac{A_{L}}{S_{e f f}}=T_{e q} \frac{A_{L}}{S_{e f f}}=r_{S} T_{e q}$

Being $A_{L}$ and $A_{C}$ the thermal losses effective area and the absorbing area, respectively.

Applying conventional definition of thermal performance (eq. (2b)), and using the proposed definition for the equivalent temperature we obtain:

$\eta_{t h}=(\tau \alpha)_{n}-U_{L}^{o} T_{e q}^{o}=(\tau \alpha)_{n}-U_{L}^{o} T_{e q} r_{S}$

We calculated the $\mathrm{r}_{\mathrm{S}}$ value using the proposed model for effective surface [12], obtaining $S_{\text {eff }}=0.3615 \mathrm{~m}^{2}$, while thermal losses effective area is of $1.2422 \mathrm{~m}^{2}$; therefore:

$r_{S}=\frac{A_{L}}{S_{\text {eff }}}=\frac{1.2422}{0.3615}=3.4362$

Now comparing equations (2b) and (24), we have:

$U_{L}^{o} r_{s}=U_{L} \rightarrow U_{L}^{o}=\frac{U_{L}}{r_{S}}=\frac{16.75}{3.4362}=4.875$

This matches perfectly the experimental result.

Optical efficiency can also be modified if we apply a correction factor to the $(\tau \alpha)$ parameter. Because $\alpha$ depends on the angle of incidence as well as on the radiation wavelength, the absortivity

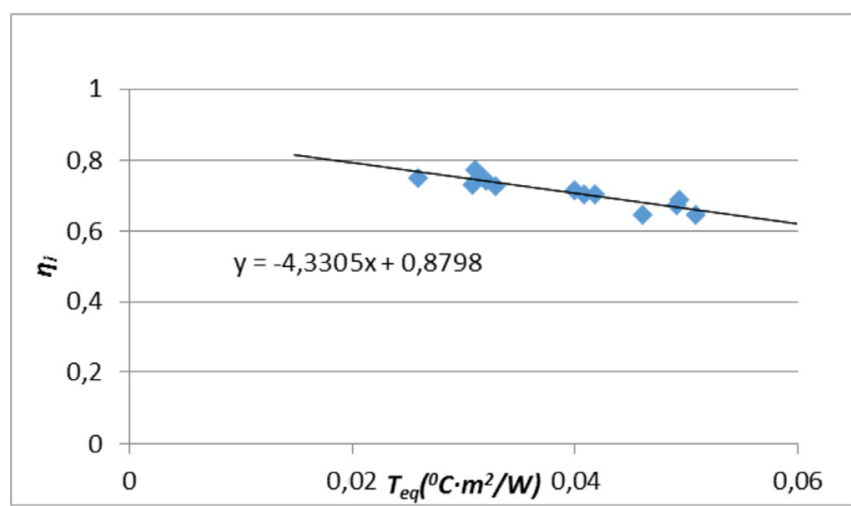

Fig. 11. a. Thermal performance of semispherical solar collector (2nd test): Collector1, (b)Thermal performance of semispherical solar collector (2nd test): Collector2. 


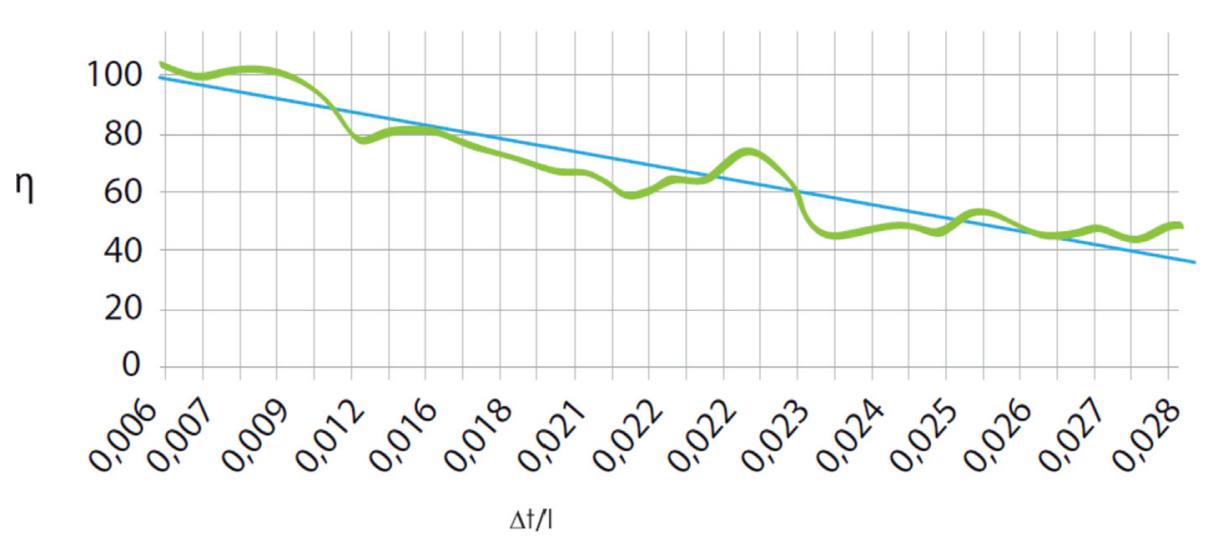

Fig. 12. Thermal performance of semispherical solar collector (manufacturer's data).

should be expressed as $\alpha=\alpha(\theta, \lambda)$. Considering direct and diffuse radiation correspond to a specific wavelength, $\lambda_{\mathrm{b}}$ and $\lambda_{\mathrm{d}}$, respectively, and taking into account the absorber area covered by each component, we have:

$\alpha(\theta, \lambda)=\frac{\alpha_{b} S_{b}+\alpha_{d} S_{d}}{A_{c}}$

$\alpha_{b}$ and $\alpha_{d}$ are the absortivity for $\lambda_{b}$ and $\lambda_{d}$, respectively.

If we assume the hypothesis that optical efficiency equals $100 \%$ as proposed by the manufacturer, $\tau=\alpha=1$. As these parameters are currently defined for normal incidence, we can establish $\alpha_{b}=\alpha=1$. On the other hand, if we consider the effects of direct radiation on the absortivity is of much higher influence than diffuse, $S_{d}=A_{c}-S_{b}$; therefore, eq. (27) transforms in:

$\alpha=r_{S b}\left(1-\alpha_{d}\right)+\alpha_{d}$

The ratio $r_{S b}=\left(S_{b} / A_{c}\right)$ is taken from a previous work [18] being $r_{S b}=0.2691$, and the $\alpha_{d}$ coefficient is considered at an angle of incidence equals to the latitude of location, in our case: $\alpha_{d}=0.7614$. Using data we have $\alpha=0.8256$ a value which is coincident with the experimental one.

\section{Conclusions}

Application of conventional expression for thermal performance in semispherical collectors leads to an overestimation of the overall thermal losses coefficient and of the optical efficiency. The overestimation in the overall thermal losses coefficient can be corrected using a correction factor that takes into accounts the ratio of thermal losses area to collector's effective surface.

A new definition for the "equivalent temperature", $T_{\text {eq }}$, has been proposed. This new definition allows the use of the classical expression structure, but replacing the former coefficients for the new ones. The use of the modified expression gives real thermal losses coefficient. Optical efficiency has also been corrected using an expression that takes into account the contribution of direct and diffuse radiation to the absortivity.

Calculated values using the proposed algorithms match experimental values, proving the validity of the new expressions.

\section{References}

[1] Theoretical calculation of energy received by semi-spherical solar collector, I. Pelēce, U. Iljins, and I. Ziemelis, Agron. Res. 6 (Special issue) (2008) 263-269.

[2] Water heating effectiveness of semi-spherical solar collector, ilze pelece, imants ziemelis, in: Renewable Energy And Energy Efficiency Proceedings of the International Scientific Conference, Latvia_Univ_Agriculture_Reee_conference, 2012.

[3] Semi-Spherical Solar Collector For Water Heating, 2010. Ilze Pelece, ENGINEERING FOR RURAL DEVELOPMENT Jelgava, 27.-28.05.

[4] Solar Engineering of Thermal Processes John A. Duffie, William A. Beckman, third ed., John Wiley \& Sons, 2016.

[5] ISO 9806, Solar Energy - Solar Thermal Collectors - Test Methods, 2017.

[6] Toward a Standard Testing Methodology for Solar Thermal Collectors with Variable-Geometry: the Direct Radiation Incidence Angle Modifier Issue vol. 121, November 2015. Fabienne Sallaberry, Ramon Pujol-Nadal, Alberto García de Jalón and Víctor Martínez-Moll. Solar Energy.

[7] Study and Characterization of Semi-spherical Solar Thermal Systems of New Generation, 2012. Ana Sofía Morillo Candás. Master Thesis. Applied Physics Master. UCM.

[8] Study and Characterization of a Spherical Solar Collector for of Water Production, 2011. Ignacio Santos Gallego. Master Thesis. Applied Physics Master UCM.

[9] Optical study and characterization of the solar radiation onto spherical solar collectors, Leticia Bragado Alonso, Master Thesis. Applied Physics Master, UCM, 2013.

[10] Project EUFRAT. Final Scientific Report. Solar Energy Research and Development in the European Community. Solar Radiation Data, 1990. Edited by Bernard Bourges. January.

[11] A correlation model to compute the incidence angle modifier and to estimate its effects on collectible solar radiation. C. Armenta-Deu and Boris Lukac. Renewable Energy. Vol. 1. Issues 5-6. 1991.

[12] An Introduction to Solar Radiation, Muhammad Iqbal. Academic Press, 1983.

[13] C. Armenta-Déu, Mathematical Model to Determine Interception Area for Direct Solar Radiation in Semi-spherical Collectors, October 2018. Renewable Energy (under revision).

[14] Active Solar Collectors and Their Applications, Ari Rabl. Oxford University Press, 1985.

[15] Study and Characterization of New Generation Semispherical Thermal Collectors, 2012. Ana Sofía Morillo Candás. Applied Physics Technical Report. UCM. September.

[16] Semispherical Solar Collector. Technical Data Bulletin. Bubblesun S.L.

[17] An Introduction to Error Analysis" by John R. Taylor (ISBN 978-0-935702-75$0)$.

[18] Development and Analysis of Characteristics Parameters for the Evaluation of Semispherical Solar Collector's Thermal Performance, June 2013. Rodrigo Varela. Applied Physics Master. Master Thesis. 